\title{
Is the Arthroscopic "Multiple Pulled Suture" Technique a Good Solution for Large or Comminuted Bony Bankart Lesions
}

\author{
Doo-Sup Kim \\ Department of Orthopedic Surgery, Yonsei University Wonju College of Medicine, Wonju, Korea
}

Numerous surgical procedures have been developed since restoration of the anterior-inferior glenoid was raised as an important factor for restoring the stability of the shoulder joint ${ }^{1)}$ after acute or chronic shoulder dislocation. ${ }^{2-5)}$ The existence of numerous methods is evidence that there is still no established method for surgery.

In the 2000s, new methods utilizing suture anchors were designed. However, this technique could not provide sufficient contact area for the fractured fragment because the suture crossed the surface of the fracture and the bony fragment could tilt because of the one-point fixation. To solve this problem, Millett et al. .) developed the "bony Bankart bridge" procedure; however, this method was technically too difficult. Bauer et al." later introduced the transglenoid technique for arthroscopic repair, which is simpler than techniques using suture anchors, but less accurate due to suture fixation on the fascia of the infraspinatus muscle.

Lee et al. ${ }^{8)}$ modified the transglenoid technique to devise a so-called pulled sutures technique.

Their study investigated the multiple pulled suture (MPS) technique in 10 patients with bony Bankart lesions and found excellent clinical and radiological results. Moreover, indirect fixation induced by ligamentotaxis was theoretically described in the discussion section of their study. In their study, 3-dimensionalcomputed tomography scans performed at 3 months postoperation showed union in all patients. However, it is not known whether this method will have the same effect on all large or comminuted bony Bankart lesions. As stated in their paper, if the condition of the capsulolabral complex and the periosteum were poor, the arthroscopic MPS technique could not be performed because it is difficult to induce a ligamentotaxis effect when periosteum damage is severe. Therefore, it is very important to confirm the condition of soft tissues around the bony fragment including the periosteum, using magnetic resonance arthrogram.

The MPS technique involves the fixation of MPS onto the 2 or 10 o'clock position on the glenoid with one Pushlock (Arthrex) anchor, a knotless suture anchor. This technique seems to have advantages, such as being simple and more economical than other conventional surgery methods that use an average of four or more suture anchors. Conversely, this method relies on one knotless suture anchor to sustain all of the tension. If this fixation fails, adverse results are obvious. Therefore, the quality of the glenoid bone should be guaranteed so that it can withstand this one suture anchor with excessive tension. In addition, the quality of the soft tissue should be ensured because MPS will be performed on the labroligamentous complex.

In this study, 7 out of 10 cases were Bigliani type I lesions and 7 out of 10 cases were first time dislocations. These factors suggest that most patients in this study had better bone quality and capsulolabral complex quality when compared to patients with chronic dislocations, resulting in solid results. Accordingly, it is important to check the patient's history to confirm whether the dislocation is acute or chronic when considering this technique. ${ }^{8)}$

As the authors pointed out in their paper, more research subjects will be needed in the future and additional biomechanical testing should be conducted utilizing cadavers to provide more robust results.

\section{References}

1. Burkhart SS, De Beer JF. Traumatic glenohumeral bone defects and their relationship to failure of arthroscopic Bankart repairs: significance of the inverted-pear glenoid and the humeral engaging Hill-Sachs lesion. Arthroscopy. 2000;16(7):677-94.

2. Sugaya H, Kon Y, Tsuchiya A. Arthroscopic repair of glenoid fractures using suture anchors. Arthroscopy. 2005;21(5):635.

\section{Correspondence to: Doo-Sup Kim}

Department of Orthopedic Surgery, Yonsei University Wonju College of Medicine, 20 Ilsan-ro, Wonju 26426, Korea

Tel: +82-33-741-0114, Fax: +82-33-741-1357, E-mail: dskim1974@hanmail.net

Editorial does not need an IRB approval.

Financial support: None. Conflict of interests: None. 
3. Cameron SE. Arthroscopic reduction and internal fixation of an anterior glenoid fracture. Arthroscopy. 1998;14(7):743-6.

4. Porcellini G, Paladini P, Campi F, Paganelli M. Long-term outcome of acute versus chronic bony Bankart lesions managed arthroscopically. Am J Sports Med. 2007;35(12):2067-72.

5. Sugaya H, Moriishi J, Kanisawa I, Tsuchiya A. Arthroscopic osseous Bankart repair for chronic recurrent traumatic anterior glenohumeral instability. Surgical technique. J Bone Joint Surg Am. 2006;88 Suppl 1 Pt 2:159-69.
6. Millett PJ, Horan MP, Martetschläger F. The "bony Bankart bridge" technique for restoration of anterior shoulder stability. Am J Sports Med. 2013;41(3):608-14.

7. Bauer T, Abadie O, Hardy P. Arthroscopic treatment of glenoid fractures. Arthroscopy. 2006;22(5):569.e1-6.

8. Lee BI, Kim BM, Kho DW, Kim HJ. Clinical results of the arthroscopic "multiple pulled suture" technique for large or comminuted bony Bankart lesion. Clin Shoulder Elbow. 2017; 20(3):138-46. 